\title{
Compactness of Composition Operators from the p-Bloch Space to the q-Bloch Space on the Classical Bounded Symmetric Domains
}

\section{Jianbing Su*, Huijuan Li, Xingxing Miao, Rui Wang}

School of Mathematics and Statistics, Jiangsu Normal University, Xuzhou, China

Email: sujb@jsnu.edu.cn

Received 31 October 2014; revised 30 November 2014; accepted 12 December 2014

Copyright (C) 2014 by authors and Scientific Research Publishing Inc.

This work is licensed under the Creative Commons Attribution International License (CC BY).

http://creativecommons.org/licenses/by/4.0/

(c) (;) Open Access

\section{Abstract}

In this paper, we introduce the weighted Bloch spaces $\beta^{p}\left(\mathfrak{R}_{I}(m, n)\right)$ on the first type of classical bounded symmetric domains $\mathfrak{R}_{I}(m, n)$, and prove the equivalence of the norms $\|f\|_{1, p}$ and $\|f\|_{2, p^{+}(m+n) / 2}$. Furthermore, we study the compactness of composition operator $C_{\phi}$ from $\beta^{p}\left(\Re_{I}(\boldsymbol{m}, n)\right)$ to $\beta^{q}\left(\mathfrak{R}_{I}(\boldsymbol{m}, \boldsymbol{n})\right)$, and obtain a sufficient and necessary condition for $C_{\phi}: \beta^{p}\left(\Re_{I}(m, n)\right) \rightarrow \beta^{q}\left(\Re_{I}(m, n)\right)$ to be compact.

\section{Keywords}

Bloch Space, Classical Bounded Symmetric Domains, Composition Operators, Compactness, Bergman Metric

\section{Introduction}

Let $\Omega$ be a bounded homogeneous domain in $\mathbb{C}^{n}$. The class of all holomorphic functions on $\Omega$ will be denoted by $H(\Omega)$. For $\phi$ a holomorphic self-map of $\Omega$ and $f \in H(\Omega)$, the composition $f \circ \phi$ is denoted by $C_{\phi} f$, and $C_{\phi}$ is called the composition operator with symbol $\phi$.

The composition operators as well as related operators known as the weighted composition operators between

\footnotetext{
${ }^{*}$ Corresponding author.
}

How to cite this paper: Su, J.B., Li, H.J., Miao, X.X. and Wang, R. (2014) Compactness of Composition Operators from the p-Bloch Space to the q-Bloch Space on the Classical Bounded Symmetric Domains. Advances in Pure Mathematics, 4, 649-664. http://dx.doi.org/10.4236/apm.2014.412074 
the weighted Bloch spaces were investigated in [1] [2] in the case of the unit disk, and in [3]-[7] for the case of the unit ball. The study of the weighted composition operators from the Bloch space to the Hardy space $H^{\infty}$ was carried out in [8] [9] for the unit ball. Characterizations of the boundedness and the compactness of the composition operators and the weighted ones between the Bloch spaces were given in [10]-[12] for the polydisc case, and in [13]-[18] for the case of the bounded symmetric domains. Furthermore, we will give some results about the composition operators for the case of the weighted Bloch space on the bounded symmetric domains.

In 1930s all irreducible bounded symmetric domains were divided into six types by E. Cartan. The first four types of irreducible domains are called the classical bounded symmetric domains, the other two types, called exceptional domains, consist of one domain each (a 16 and 27 dimensional domain).

The first three types of classical bounded symmetric domains can be expressed as follows [19]:

$$
\mathfrak{R}_{I}(m, n)=\left\{Z: Z \text { is an } m \times n \text { complex matrix, } I_{m}-Z \bar{Z}^{\mathrm{T}}>0\right\},
$$

where $m \leq n$ and $I_{m}$ is the $m \times m$ identity matrix, $Z^{\mathrm{T}}$ is the transpose of $Z$;

$$
\begin{gathered}
\mathfrak{R}_{I I}(p)=\left\{Z: Z \text { is a } p \times p \text { symmetric matrix, } Z=Z^{\mathrm{T}}, I_{p}-Z \bar{Z}>0\right\} \\
\mathfrak{R}_{I I I}(q)=\left\{Z: Z \text { is a } q \times q \text { antisymmetric matrix, } Z=-Z^{\mathrm{T}}, I_{q}+Z \bar{Z}>0\right\} .
\end{gathered}
$$

Let $A=\left(a_{i j}\right)_{m \times n}$ and $B=\left(b_{k l}\right)_{s \times t}$. The Kronecker product $A \times B$ of $A$ and $B$ is defined as the $m s \times n t$ matrix $C=\left(c_{i k j l}\right)$ such that the element at the $i k$-th row and $j l$-th column $c_{i k j l}=a_{i j} b_{k l} \quad$ [19]. Then the Bergman metric of $\mathfrak{R}_{I}(m, n)$ is as follows (see [19]):

$$
H_{Z}(u, u)=(m+n) u\left(I-Z \bar{Z}^{\mathrm{T}}\right)^{-1} \times\left(I-\bar{Z}^{\mathrm{T}} Z\right)^{-1} \bar{u}^{\mathrm{T}},
$$

where $u=\left(u_{11}, \cdots, u_{1 n}, \cdots, u_{m 1}, \cdots, u_{m n}\right)$ is a complex vector, $\bar{u}^{\mathrm{T}}$ is the conjugate transpose of $u$, and $Z=\left(z_{i j}\right)_{m \times n} \in \mathfrak{R}_{I}(m, n)$.

Following Timoney's approach (see [18]), a holomorphic function $f$ is in the Bloch space $\beta\left(\Re_{I}(m, n)\right)$, if

$$
\|f\|_{\beta}=\sup _{Z \in \mathfrak{R}_{I}(m, n)} Q_{f}(Z)<\infty .
$$

Now we define a holomorphic function $f$ to be in the p-Bloch space $\beta^{p}\left(\Re_{I}(m, n)\right)$, if

$$
\|f\|_{\beta^{p}}=\sup _{Z \in \Re_{I}(m, n)} \operatorname{det}\left(I-Z \bar{Z}^{\mathrm{T}}\right)^{p} Q_{f}(Z)<\infty,
$$

where

$$
\begin{gathered}
Q_{f}(Z)=\sup \left\{\frac{|\nabla f(Z) u|}{H_{Z}^{1 / 2}(u, u)}: u \in \mathbb{C}^{m n}-\{0\}\right\}, \\
\nabla f(Z)=\left(\frac{\partial f}{\partial z_{11}}(Z), \cdots, \frac{\partial f}{\partial z_{1 n}}(Z), \cdots, \frac{\partial f}{\partial z_{m 1}}(Z), \cdots, \frac{\partial f}{\partial z_{m n}}(Z)\right) .
\end{gathered}
$$

We can prove that $\beta^{p}\left(\mathfrak{R}_{I}(m, n)\right)$ is a Banach space with norm $\|f\|_{1, p}=|f(0)|+\|f\|_{\beta^{p}}$ which is similar with the case on $\beta^{\alpha}\left(B^{n}\right)$.

Let $\phi=\left(\phi_{i j}\right)_{m \times n}$ be a holomorphic self-map of $\mathfrak{R}_{I}(m, n)$. We are concerned here with the question of when $C_{\phi}: \beta^{p}\left(\mathfrak{R}_{I}(m, n)\right) \rightarrow \beta^{q}\left(\mathfrak{R}_{I}(m, n)\right)$ will be a compact operator.

Let $\operatorname{diag}\left(d_{1}, \cdots, d_{n}\right)$ denote a diagonal matrix with diagonal elements $d_{1}, \cdots, d_{n}$. In this work,we shall denote by $C$ a positive constant, not necessarily the same on each occurrence.

In Section 2, we prove the equivalence of the norms defined in this paper and in [20].

In Section 3, we state several auxiliary results most of which will be used in the proofs of the main results.

Finally, in Section 4, we establish the main result of the paper. We give a sufficient and necessary condition for the composition operator $\mathrm{C}_{\phi}$ from the p-Bloch space $\beta^{p}\left(\mathfrak{R}_{I}(m, n)\right)$ to the q-Bloch space $\beta^{q}\left(\mathfrak{R}_{I}(m, n)\right)$ to be compact, where $p \geq 0$ and $q \geq 0$. Specifically,we prove the following result: 
Theorem 1.1. Let $\phi$ be a holomorphic self-map of $\mathfrak{R}_{I}(m, n)$. Then $C_{\phi}: \beta^{p}\left(\mathfrak{R}_{I}(m, n)\right) \rightarrow \beta^{q}\left(\mathfrak{R}_{I}(m, n)\right)$ is compact if and only if, for every $\varepsilon>0$, there exists $a \quad \delta>0$ such that

$$
\frac{\operatorname{det}\left(I-Z \bar{Z}^{\mathrm{T}}\right)^{q}}{\operatorname{det}\left(I-\phi(Z) \overline{\phi(Z)}^{\mathrm{T}}\right)^{p}} \frac{H_{\phi(Z)}^{1 / 2}(J \phi(Z) u, J \phi(Z) u)}{H_{Z}^{1 / 2}(u, u)}<\varepsilon,
$$

for all $u \in \mathbb{C}^{m n}-\{0\}$ whenever $\operatorname{dist}\left(\phi(Z), \partial \mathfrak{R}_{I}\right)<\delta, \quad Z \in \mathfrak{R}_{I}(m, n)$.

The compactness of the composition operators for the weighted Bloch space on the bounded symmetric domains of $\mathfrak{R}_{I I}(p), \mathfrak{R}_{I I I}(q)$ is similar with the case of $\mathfrak{R}_{I}(m, n)$; we omit the details.

\section{The Equivalence of the Norms}

Denote [20] $\|f\|_{2, \beta^{p}}=\sup _{Z \in \Re_{I}(m, n)} \operatorname{det}\left(I-Z \bar{Z}^{\mathrm{T}}\right)^{p}|\nabla f(Z)|$ and $\|f\|_{2, p}=|f(0)|+\|f\|_{2, \beta^{p}}$.

Lemma 2.1. (Bloomfield-Watson) [21] Let $A=\left(a_{i j}\right) \geq 0$ be an $m \times m$ Hermitian matrix. Then

$$
\operatorname{det}\left(\bar{B}^{\mathrm{T}} A B\right) \leq \operatorname{det} A
$$

where $B$ is any $m \times n$ matrix and satisfies $B \bar{B}^{\mathrm{T}}=I$.

Theorem 2.1. $\|f\|_{1, p}$ and $\|f\|_{2, p+(m+n) / 2}$ are equivalent.

Proof. The metric matrix of $\mathfrak{R}_{I}(m, n)$ is

$$
T(Z, Z)=(m+n)\left(I_{m}-Z \bar{Z}^{\mathrm{T}}\right)^{-1} \times\left(I_{n}-\bar{Z}^{\mathrm{T}} Z\right)^{-1}
$$

For any $Z \in \mathfrak{R}_{I}(m, n)$, let $\varphi_{Z} \in \operatorname{Aut}\left(\mathfrak{R}_{I}(m, n)\right)$ with $\varphi_{Z}(Z)=0$. Then

$$
T(Z, Z)=\left(\left(J \varphi_{Z}\right)(Z)\right)^{\mathrm{T}} T(0,0) \overline{\left(J \varphi_{Z}\right)(Z)}=(m+n)\left(\left(J \varphi_{Z}\right)(Z)\right)^{\mathrm{T}} \overline{\left(J \varphi_{Z}\right)(Z)}
$$

Denote $\varphi_{Z}^{-1}=\psi_{Z}$ then $\psi_{Z}(0)=Z, J \psi_{Z}=\left(J \varphi_{Z}\right)^{-1}$ and

Thus

$$
\left.T^{-1}(Z, Z)=\frac{1}{m+n} \overline{\left(J \varphi_{Z}\right)(Z)}\right)^{-1}\left(\left(J \varphi_{Z}\right)(Z)^{\mathrm{T}}\right)^{-1}=\frac{1}{m+n} \overline{\left(J \psi_{Z}\right)(0)}\left(J \psi_{Z}\right)(0)^{\mathrm{T}} .
$$

$$
\overline{\nabla f(Z)} T^{-1}(Z, Z)(\nabla f(Z))^{\mathrm{T}}=\frac{1}{m+n} \overline{\nabla f(Z)\left(J \psi_{Z}\right)(0)}\left(\nabla f(Z)\left(J \psi_{Z}\right)(0)\right)^{\mathrm{T}}=\frac{1}{m+n}\left|\nabla\left(f \circ \psi_{Z}\right)(0)\right|^{2} .
$$

Hence

$$
\begin{aligned}
\operatorname{det}\left(I-Z \bar{Z}^{\mathrm{T}}\right)^{p} Q_{f \circ \psi_{Z}}(0) & =\operatorname{det}\left(I-Z \bar{Z}^{\mathrm{T}}\right)^{p} \sup \left\{\frac{\left|\nabla\left(f \circ \psi_{Z}\right)(0) u\right|}{H_{0}^{1 / 2}(u, u)}: u \in \mathbb{C}^{m n}-\{0\},|u|=1\right\} \\
& =\frac{1}{\sqrt{m+n}} \operatorname{det}\left(I-Z \bar{Z}^{\mathrm{T}}\right)^{p} \sup \left\{\left|\nabla\left(f \circ \psi_{Z}\right)(0) u\right|: u \in \mathbb{C}^{m n}-\{0\},|u|=1\right\} \\
& =\operatorname{det}\left(I-Z \bar{Z}^{\mathrm{T}}\right)^{p}\left\{\overline{\nabla f(Z) T^{-1}}(Z, Z)(\nabla f(Z))^{\mathrm{T}}\right\}^{1 / 2} .
\end{aligned}
$$

Furthermore,

$$
\begin{aligned}
\|f\|_{\beta^{p}} & =\sup _{Z \in \Re_{I}(m, n)} \operatorname{det}\left(I-Z \bar{Z}^{\mathrm{T}}\right)^{p} Q_{f}(Z) \\
& =\sup _{Z \in \Re_{I}(m, n)} \operatorname{det}\left(I-Z \bar{Z}^{\mathrm{T}}\right)^{p}\left\{\overline{\nabla f(Z)} T^{-1}(Z, Z)(\nabla f(Z))^{\mathrm{T}}\right\}^{1 / 2} .
\end{aligned}
$$

Since

$$
\begin{aligned}
\overline{\nabla f(Z)} T^{-1}(Z, Z)(\nabla f(Z))^{\mathrm{T}} & \leq|\nabla f(Z)|^{2} \operatorname{det} T^{-1}(Z, Z) \\
& =(m+n)^{-1}|\nabla f(Z)|^{2} \operatorname{det}\left(I-Z \bar{Z}^{\mathrm{T}}\right)^{m+n} .
\end{aligned}
$$


Thus

$$
\|f\|_{\beta^{p}} \leq \frac{1}{\sqrt{m+n}}\|f\|_{2, \beta}{ }^{p+\frac{m+n}{2}} .
$$

For

$$
\begin{aligned}
\operatorname{det}\left(I-Z \bar{Z}^{\mathrm{T}}\right)^{p} Q_{f}(Z) & =\operatorname{det}\left(I-Z \bar{Z}^{\mathrm{T}}\right)^{p} \sup \left\{\frac{|\nabla f(Z) u|}{\left\{u T(Z, Z) \bar{u}^{\mathrm{T}}\right\}^{1 / 2}}: u \in \mathbb{C}^{m n}-\{0\},|u|=1\right\} \\
& \geq \operatorname{det}\left(I-Z \bar{Z}^{\mathrm{T}}\right)^{p}\left\{\operatorname{det} T^{-1}(Z, Z)\right\}^{1 / 2}|\nabla f(Z)| \\
& =\frac{1}{\sqrt{m+n}} \operatorname{det}\left(I-Z \bar{Z}^{\mathrm{T}}\right)^{p+\frac{m+n}{2}}|\nabla f(Z)|
\end{aligned}
$$

then we have

$$
\|f\|_{\beta^{p}} \geq \frac{1}{\sqrt{m+n}}\|f\|_{2, \beta}^{p+\frac{m+n}{2}} .
$$

Combining (2.2) and (2.3),

$$
\|f\|_{\beta^{p}}=\frac{1}{\sqrt{m+n}}\|f\|_{2, \beta}^{p+\frac{m+n}{2}} .
$$

Next,

$$
\|f\|_{1, p}=|f(0)|+\|f\|_{\beta^{p}} \leq|f(0)|+\|f\|_{2, \beta}^{p+\frac{m+n}{2}}=\|f\|_{2, p+\frac{m+n}{2}}
$$

and

$$
\|f\|_{2, p+\frac{m+n}{2}}=|f(0)|+\|f\|_{2, \beta}{ }^{p+\frac{m+n}{2}} \leq \sqrt{m+n}\left(|f(0)|+\|f\|_{\beta^{p}}\right)=\sqrt{m+n}\|f\|_{1, p}
$$

Therefore, the proof is completed.

\section{Some Lemmas}

Here we state several auxiliary results most of which will be used in the proof of the main result.

Lemma 3.1. [18] Let $\mathcal{D} \subset \mathbb{C}^{N}$ be a bounded homogeneous domain. Then there exists a constant $C$, depending only on $\mathcal{D}$, such that

$$
H_{\phi(z)}(J \phi(z) u, J \phi(z) u) \leq C H_{z}(u, u)
$$

for each $z \in \mathcal{D}$ whenever $\phi$ holomorphically maps $\mathcal{D}$ into itself. Here $H_{z}(u, u)$ denotes the Bergman metric on $\mathcal{D}, J \phi(z)=\left(\frac{\partial \phi_{l}(z)}{\partial z_{k}}\right)_{1 \leq l, k \leq N}$ denotes the Jacobian matrix of $\phi$.

Lemma 3.2. Let $\phi$ be a holomorphic self-map of $\mathfrak{R}_{I}(m, n)$ and $K$ a compact subset of $\mathfrak{R}_{I}(m, n)$. Then there exists a constant $C>0$ such that

$$
\frac{\operatorname{det}\left(I-Z \bar{Z}^{\mathrm{T}}\right)^{q}}{\operatorname{det}\left(I-\phi(Z) \overline{\phi(Z)^{\mathrm{T}}}\right)^{p}} \frac{H_{\phi(Z)}^{1 / 2}(J \phi(Z) u, J \phi(Z) u)}{H_{Z}^{1 / 2}(u, u)} \leq C
$$

for all $u \in \mathbb{C}^{m n}-\{0\}$ whenever $\phi(Z) \in K$.

Proof. For $\delta \in(0,1)$, let $E_{W}^{\delta}:=\left\{W \in \mathfrak{R}_{I}(m, n): \operatorname{dist}\left(W, \partial \mathfrak{R}_{I}\right) \geq \delta\right\}$.

For any compact $K \subset \mathfrak{R}_{I}(m, n)$, there exists a constant $\delta \in(0,1)$ such that $K \subset E_{W}^{\delta}$. Then there exists $M \in(0,1)$ such that $\operatorname{det}\left(I-\phi(Z) \overline{\phi(Z)}^{\mathrm{T}}\right)>M$, whenever $\phi(Z) \in K$. 
Thus

$$
\frac{\operatorname{det}\left(I-Z \bar{Z}^{\mathrm{T}}\right)^{q}}{\operatorname{det}\left(I-\phi(Z) \overline{\phi(Z)}^{\mathrm{T}}\right)^{p}}<\frac{1}{M^{p}} .
$$

Combining Lemma 3.1 with (3.3) shows that (3.2) holds.

Lemma 3.3. (Hadamard) [21] Let $A=\left(a_{i j}\right) \geq 0$ be an $n \times n$ Hermitian matrix. Then

$$
\operatorname{det} A \leq \prod_{i=1}^{n} a_{i i}
$$

and equality holds if and only if $A$ is a diagonal matrix.

Lemma 3.4. Let $Z=\left(z_{k l}\right)_{m \times n} \in \Re_{I}(m, n)$. Then

$$
\operatorname{det}\left(I_{m}-Z \bar{Z}^{\mathrm{T}}\right) \leq \prod_{i=1}^{m}\left(1-\left|z_{i i}\right|^{2}\right) \text {. }
$$

Proof. For any $Z \in \mathfrak{R}_{I}(m, n)$, we have $I_{m}-Z \bar{Z}^{\mathrm{T}}=\left(\delta_{s t}-\sum_{j=1}^{n} z_{s j} \bar{z}_{t j}\right)_{1 \leq s, t \leq m}>0$.

Thus we have $0<1-\sum_{j=1}^{n}\left|z_{i j}\right|^{2}<1, \quad i=1,2, \cdots, m$.

It follows from Lemma 3.3 that $\operatorname{det}\left(I_{m}-Z \bar{Z}^{\mathrm{T}}\right) \leq \prod_{i=1}^{m}\left(1-\sum_{j=1}^{n}\left|z_{i j}\right|^{2}\right) \leq \prod_{i=1}^{m}\left(1-\left|z_{i i}\right|^{2}\right)$.

Lemma 3.5. Let $\Re_{I}(m, n)$ be a classical bounded symmetric domain, and $T(z, z)$ denote its metric matrix. Then a holomorphic function $f$ on $\mathfrak{R}_{I}(m, n)$ is in $\beta^{p}\left(\Re_{I}(m, n)\right)$ if and only if

$$
\sup _{Z \in \Re_{I}(m, n)} \operatorname{det}\left(I-Z \bar{Z}^{\mathrm{T}}\right)^{p}\left\{\nabla f(Z) T^{-1}(Z, Z) \overline{\nabla f(Z)}^{\mathrm{T}}\right\}^{1 / 2}<\infty .
$$

If (3.6) holds, then

$$
\|f\|_{\beta^{p}} \leq \sup _{Z \in \Re_{I}(m, n)} \operatorname{det}\left(I-Z \bar{Z}^{\mathrm{T}}\right)^{p}\left\{\nabla f(Z) T^{-1}(Z, Z) \overline{\nabla f(Z)}^{\mathrm{T}}\right\}^{1 / 2} .
$$

Proof. We can get the conclusion by the process of the proof on Theorem 2.1.

Lemma 3.6. [18] Let

$$
\begin{aligned}
& P=U\left(\operatorname{diag}\left(\lambda_{1}, \lambda_{2}, \cdots, \lambda_{m}\right), 0\right) V \in \mathfrak{R}_{I}, \\
& Q=U \operatorname{diag}\left(\frac{1}{\sqrt{1-\lambda_{1}^{2}}}, \frac{1}{\sqrt{1-\lambda_{2}^{2}}}, \cdots, \frac{1}{\sqrt{1-\lambda_{m}^{2}}}\right) \bar{U}^{\mathrm{T}}, \\
& R=\bar{V}^{\mathrm{T}} \operatorname{diag}\left(\frac{1}{\sqrt{1-\lambda_{1}^{2}}}, \frac{1}{\sqrt{1-\lambda_{2}^{2}}}, \cdots, \frac{1}{\sqrt{1-\lambda_{m}^{2}}}, 1, \cdots, 1\right) V,
\end{aligned}
$$

where $U_{m \times m}$ and $V_{n \times n}$ are unitary matrices and $0 \leq \lambda_{m} \leq \cdots \leq \lambda_{2} \leq \lambda_{1}<1$.

Denote $\Phi_{P}(Z)=Q(P-Z)\left(I_{n}-\bar{P}^{\mathrm{T}} Z\right)^{-1} R^{-1}, Z \in \mathfrak{R}_{I}(m, n)$. Then

(1) $\Phi_{P}(Z) \in \operatorname{Aut}\left(\Re_{I}(m, n)\right)$;

(2) $\left(\Phi_{P}\right)^{-1}=\Phi_{P}$;

(3) $\Phi_{P}(0)=P$ and $\Phi_{P}(P)=0$;

(4) $\left.\mathrm{d} \Phi_{P}(Z)\right|_{Z=P}=-Q \mathrm{~d} Z R$ and $\left.\mathrm{d} \Phi_{P}(Z)\right|_{Z=0}=-Q^{-1} \mathrm{~d} Z R^{-1}$ for $Z \in \mathfrak{R}_{I}(m, n)$; 
(5) $\Phi_{P}(Z)=Q^{-1}\left(I_{m}-Z \bar{P}^{\mathrm{T}}\right)^{-1}(P-Z) R$ for $Z \in \mathfrak{R}_{I}(m, n)$;

(6) $\left(I_{m}-Z \bar{P}^{\mathrm{T}}\right) Q\left(I_{m}-\Phi_{P}(Z){\bar{\Phi} \bar{P}(Z)}^{\mathrm{T}}\right) \bar{Q}^{\mathrm{T}}\left(I_{m}-P \bar{Z}^{\mathrm{T}}\right)=I_{m}-Z \bar{Z}^{\mathrm{T}}$ for $Z \in \mathfrak{R}_{I}(m, n)$.

Lemma 3.7. $C_{\phi}: \beta^{p}\left(\Re_{I}(m, n)\right) \rightarrow \beta^{q}\left(\Re_{I}(m, n)\right)$ is compact if and only if $\left\|C_{\phi} f_{k}\right\|_{\beta^{q}} \rightarrow 0$ as $k \rightarrow \infty$ for any bounded sequence $\left\{f_{k}\right\}$ in $\beta^{p}\left(\Re_{I}(m, n)\right)$ that converges to 0 uniformly on compact subsets of $\Re_{I}(m, n)$.

Proof. The proof is trial by using the normal methods.

\section{Proof of Theorem 1.1}

Proof. Let $\left\{f_{k}\right\}$ be a bounded sequence in $\beta^{p}\left(\Re_{I}(m, n)\right)$ with $\left\|f_{k}\right\|_{\beta^{p}} \leq C$, and $f_{k} \rightarrow 0$ uniformly on compact subsets of $\mathfrak{R}_{I}(m, n)$.

Suppose (1.3) holds. Then for any $\varepsilon>0$, there exists a $\delta>0$, such that

$$
\frac{\operatorname{det}\left(I-Z \bar{Z}^{\mathrm{T}}\right)^{q}}{\operatorname{det}\left(I-\phi(Z) \overline{\phi(Z)}^{\mathrm{T}}\right)^{p}} \frac{H_{\phi(Z)}^{1 / 2}(J \phi(Z) u, J \phi(Z) u)}{H_{Z}^{1 / 2}(u, u)}<\frac{\varepsilon}{C}
$$

for all $u \in \mathbb{C}^{m n}-\{0\}$ whenever $\operatorname{dist}\left(\phi(Z), \partial \Re_{I}(m, n)\right)<\delta$ and $Z \in \Re_{I}(m, n)$.

By the chain rule, we have $\nabla\left(f_{k} \circ \phi\right)(Z)=\nabla\left(f_{k}\right)(\phi(Z)) J \phi(Z)$.

If $u \in \mathbb{C}^{m n}-\{0\}$ and $J \phi(Z) u=0$, then we get $\nabla\left(f_{k} \circ \phi\right)(Z) u=0$. If $u \in \mathbb{C}^{m n}-\{0\}$ and $J \phi(Z) u \neq 0$, then

$$
\frac{\nabla\left(f_{k} \circ \phi\right)(Z) u}{H_{Z}^{1 / 2}(u, u)}=\frac{\nabla\left(f_{k}\right)(\phi(Z)) J \phi(Z) u}{H_{\phi(Z)}^{1 / 2}(J \phi(Z) u, J \phi(Z) u)} \frac{H_{\phi(Z)}^{1 / 2}(J \phi(Z) u, J \phi(Z) u)}{H_{Z}^{1 / 2}(u, u)} .
$$

It follows from (4.1) and (4.2) that

$$
\begin{aligned}
\operatorname{det}\left(I-Z \bar{Z}^{\mathrm{T}}\right)^{q} Q_{C_{\phi} f_{k}}(Z)=\sup \left\{\operatorname{det}\left(I-Z \bar{Z}^{\mathrm{T}}\right)^{q} \frac{\left|\nabla\left(f_{k} \circ \phi\right)(Z) u\right|}{H_{Z}^{1 / 2}(u, u)}, u \in \mathbb{C}^{m n}-\{0\}, J \phi(Z) u \neq 0\right\} \\
\quad \leq\left\|f_{k}\right\|_{\beta^{p}} \sup \left\{\frac{\operatorname{det}\left(I-Z \bar{Z}^{\mathrm{T}}\right)^{q}}{\left.\operatorname{det}(I-\phi(Z) \overline{\phi(Z)})^{\mathrm{T}}\right)^{p}} \frac{H_{\phi(Z)}^{1 / 2}(J \phi(Z) u, J \phi(Z) u)}{H_{Z}^{1 / 2}(u, u)}, u \in \mathbb{C}^{m n}-\{0\}\right\} \\
<C \frac{\varepsilon}{C}=\varepsilon,
\end{aligned}
$$

whenever $\operatorname{dist}\left(\phi(Z), \partial \Re_{I}(m, n)\right)<\delta$ and $Z \in \mathfrak{R}_{I}(m, n)$.

On the other hand, there exists a constant $m>0$ such that

$$
\inf \left\{H_{W}^{1 / 2}(u, u):|u|=1, \operatorname{dist}\left(W, \partial \Re_{I}(m, n)\right) \geq \delta\right\}=m .
$$

So if $\operatorname{dist}\left(W, \partial \Re_{I}(m, n)\right) \geq \delta$, then

$$
\operatorname{det}\left(I-W \bar{W}^{\mathrm{T}}\right)^{p} \frac{\left|\nabla f_{k}(W) u\right|}{H_{W}^{1 / 2}(u, u)} \leq \operatorname{det}\left(I-W \bar{W}^{\mathrm{T}}\right)^{p} \frac{\left|\nabla f_{k}(W)\right|}{H_{W}^{1 / 2}(u /|u|, u /|u|)} \leq \operatorname{det}\left(I-W \bar{W}^{\mathrm{T}}\right)^{p} \frac{\left|\nabla f_{k}(W)\right|}{m} .
$$

We assume that $\left\{f_{k}\right\}$ converges to 0 uniformly on compact subsets of $\mathfrak{R}_{I}(m, n)$. By Weierstrass Theorem, it is easy to see that $\left\{\nabla f_{k}\right\}$ converges to 0 uniformly on compact subsets of $\mathfrak{R}_{I}(m, n)$. Thus, for given $\varepsilon>0$, there exists $k$ large enough such that

$$
\frac{\operatorname{det}\left(I-\phi(Z) \overline{\phi(Z)}^{\mathrm{T}}\right)^{p}\left|\nabla\left(f_{k}\right)(\phi(Z)) J \phi(Z) u\right|}{H_{\phi(Z)}^{1 / 2}(J \phi(Z) u, J \phi(Z) u)}<\frac{\varepsilon}{C}
$$


for any $u \in \mathbb{C}^{m n}-\{0\}, \quad J \phi(Z) u \neq 0$ whenever $\operatorname{dist}\left(\phi(Z), \partial \Re_{I}(m, n)\right) \geq \delta$ and $Z \in \Re_{I}(m, n)$. Then by inequalities (4.3) and (4.5) and Lemma 3.2, it follows that, for $k$ large enough,

$$
\left\|C_{\phi} f_{k}\right\|_{\beta^{q}}<\varepsilon
$$

whenever $\operatorname{dist}\left(\phi(Z), \partial \Re_{I}(m, n)\right) \geq \delta$ and $Z \in \mathfrak{R}_{I}(m, n)$.

Combining (4.4) and (4.6) shows that $\left\|C_{\phi} f_{k}\right\|_{\beta^{q}}<\varepsilon$ as $k$ large enough. So

$C_{\phi}: \beta^{p}\left(\Re_{I}(m, n)\right) \rightarrow \beta^{q}\left(\Re_{I}(m, n)\right)$ is compact.

For the converse, arguing by contradiction, suppose $C_{\phi}: \beta^{p}\left(\Re_{I}(m, n)\right) \rightarrow \beta^{q}\left(\Re_{I}(m, n)\right)$ is compact and the condition (1.3) fails. Then there exist an $\varepsilon_{0}>0$, a sequence $\left\{Z^{j}\right\}$ in $\mathfrak{R}_{I}(m, n)$ with $\phi\left(Z^{j}\right) \rightarrow \partial \Re_{I}(m, n)$ as $j \rightarrow \infty$ and a sequence $\left\{u^{j}\right\}$ in $\mathbb{C}^{m n}-\{0\}$, such that

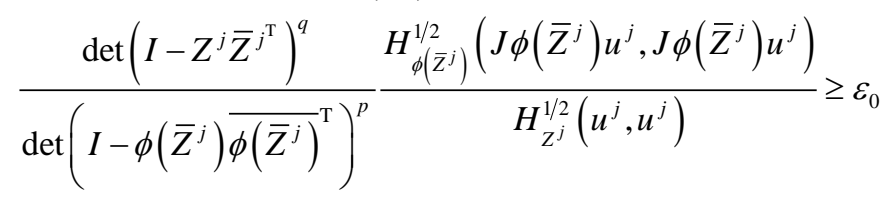

for all $j=1,2, \cdots$.

Now we will construct a sequence of functions $\left\{f_{j}\right\}$ satisfying the following three conditions :

(I) $\left\{f_{j}\right\}$ is a bounded sequence in $\beta^{p}\left(\Re_{I}(m, n)\right)$;

(II) $\left\{f_{j}\right\}$ tends to 0 uniformly on any compact subset of $\mathfrak{R}_{I}(m, n)$;

(III) $\left\|C_{\phi} f_{j}\right\|_{\beta^{q}} \nrightarrow 0$ as $j \rightarrow \infty$.

The existence of this sequence will contradict the compactness of $C_{\phi}$.

We will construct the sequence of functions $\left\{f_{j}\right\}$ according to the following four parts: A - D.

Part A: Suppose that $\phi\left(Z^{j}\right)=r_{j} E_{11}, \quad j=1,2, \cdots$

where $E_{k l}$ is the $m \times n$ matrix whose element at the $k$ th row and lth column is 1 and the other elements are 0 . Since $\phi$ maps $\mathfrak{R}_{I}(m, n)$ into itself, $0<r_{j}<1$ and $r_{j} \rightarrow 1$ as $j \rightarrow \infty$.

Denote $J \phi\left(Z^{j}\right) u^{j}$ by $w^{j}=\left(w_{11}^{j}, \cdots, w_{1 n}^{j}, w_{21}^{j}, \cdots, w_{2 n}^{j}, \cdots, w_{m 1}^{j}, \cdots, w_{m n}^{j}\right)$ Using formula (1.1), we have

$$
\begin{aligned}
H_{\phi\left(Z^{j}\right)}\left(w^{j}, w^{j}\right) & =(m+n) w^{j} \operatorname{diag}\left(\left(1-r_{j}^{2}\right)^{-1}, 1, \cdots, 1\right) \times \operatorname{diag}\left(\left(1-r_{j}^{2}\right)^{-1}, 1, \cdots, 1\right){\overline{w^{j}}}^{\mathrm{T}} \\
& =(m+n)\left[\frac{\left|w_{11}^{j}\right|^{2}}{\left(1-r_{j}^{2}\right)^{2}}+\frac{1}{1-r_{j}^{2}}\left(\sum_{l=2}^{n}\left|w_{11}^{j}\right|^{2}+\sum_{k=2}^{m}\left|w_{k 1}^{j}\right|^{2}\right)+\sum_{2 \leq k \leq m, 2 \leq l \leq n}\left|w_{k l}^{j}\right|^{2}\right] .
\end{aligned}
$$

Denote

$$
A_{j}=\frac{\left|w_{11}^{j}\right|^{2}}{\left(1-r_{j}^{2}\right)^{2}}, \quad B_{j}=\frac{1}{1-r_{j}^{2}}\left(\sum_{l=2}^{n}\left|w_{11}^{j}\right|^{2}+\sum_{k=2}^{m}\left|w_{k 1}^{j}\right|^{2}\right), \quad C_{j}=\sum_{2 \leq k \leq m, 2 \leq l \leq n}\left|w_{k l}^{j}\right|^{2},
$$

then

$$
H_{\phi\left(Z^{j}\right)}\left(w^{j}, w^{j}\right)=(m+n)\left(A_{j}+B_{j}+C_{j}\right) .
$$

We construct the sequence of functions $\left\{f_{j}\right\}$ according to the following three different cases.

Case 1. If for some $j$,

then set

$$
\max \left(B_{j}, C_{j}\right) \leq A_{j}
$$

$$
f_{j}(Z)=\frac{1}{p}\left(\frac{1}{\left(1-z_{11}\right)^{p}}-\frac{1}{\left(1-\mathrm{e}^{-a\left(1-r_{j}\right)} z_{11}\right)^{p}}\right)
$$


where $a$ is any positive number.

Case 2. If for some $j$,

$$
\max \left(A_{j}, C_{j}\right) \leq B_{j}
$$

then set

$$
f_{j}(Z)=\left(\sum_{l=2}^{n} \mathrm{e}^{-i \theta_{1 l}^{j}} z_{1 l}+\sum_{k=2}^{m} \mathrm{e}^{-i \theta_{k 1}^{j}} z_{k 1}\right)\left(\frac{1}{\left(1-z_{11}\right)^{p+1 / 2}}-\frac{1}{\left(1-\mathrm{e}^{-a\left(1-r_{j}\right)} z_{11}\right)^{p+1 / 2}}\right)
$$

where $\theta_{k l}^{j}=\arg w_{k l}^{j}$, if for some $l, w_{k l}^{j} \neq 0$ or for some $k, w_{k l}^{j}=0$, replace the corresponding term $\mathrm{e}^{-i \theta_{k l}^{j}} z_{k l}$ by 0 (the same below).

Case 3. If for some $j$,

$$
\max \left(A_{j}, B_{j}\right) \leq C_{j}
$$

then set

$$
f_{j}(Z)=\left(\sum_{2 \leq k \leq m, 2 \leq l \leq n} \mathrm{e}^{-i \theta_{k l}^{j}} z_{k l}\right)\left(\frac{1}{\left(1-z_{11}\right)^{p}}-\frac{1}{\left(1-\mathrm{e}^{-a\left(1-r_{j}\right)} z_{11}\right)^{p}}\right) .
$$

Next, we will prove that the sequences of functions $\left\{f_{j}(Z)\right\}$ defined by (4.10), (4.12) and (4.14) all satisfy the conditions (I), (II) and (III).

To begin with, we will prove the sequence of functions $\left\{f_{j}(Z)\right\}$ defined by (4.10) satisfies the three conditions. We can get that

$$
\begin{aligned}
& \left.\operatorname{det}\left(I-Z \bar{Z}^{\mathrm{T}}\right)^{p}\left\{\nabla f_{j}(Z) T^{-1}(Z, Z) \overline{\nabla f_{j}(Z)}\right\}^{\mathrm{T}}\right\}^{1 / 2} \\
& \quad=(m+n)^{-1 / 2} \operatorname{det}\left(I-Z \bar{Z}^{\mathrm{T}}\right)^{p}\left\{\left(1-\sum_{l=1}^{n}\left|z_{11}\right|^{2}\right)\left(1-\sum_{k=1}^{m}\left|z_{k 1}\right|^{2}\right)\right\}^{1 / 2}\left|\frac{1}{\left(1-z_{11}\right)^{p+1}}-\frac{\mathrm{e}^{-a\left(1-r_{j}\right)}}{\left(1-\mathrm{e}^{-a\left(1-r_{j}\right)} z_{11}\right)^{p+1}}\right| \\
& \leq(m+n)^{-1 / 2}\left(1-\left|z_{11}\right|^{2}\right)^{p}\left(1-\left|z_{11}\right|^{2}\right) \frac{2}{\left(1-\left|z_{11}\right|\right)^{p+1}} \\
& \leq 2^{p+2}(m+n)^{-1 / 2} .
\end{aligned}
$$

It follows from Lemma 3.5 that $\left\|f_{j}\right\|_{\beta^{p}} \leq C 2^{p+2}(m+n)^{-1 / 2}$.

This proves that the sequence of functions $\left\{f_{j}(Z)\right\}$ defined by (4.10) satisfies condition (I).

Let $E$ be any compact subset of $\mathfrak{R}_{I}(m, n)$. Then there exists a $\rho \in(0,1)$ such that

$$
\left|z_{11}\right| \leq \rho
$$

for any $Z=\left(z_{k l}\right)_{m \times n} \in E$. By (4.10), we have

$$
\left|f_{j}(Z)\right|=\left|\frac{1}{p} \frac{1}{\left(1-\mathrm{e}^{-a\left(1-r_{j}\right)} z_{11}\right)^{p}}\left\{\left(\frac{1-\mathrm{e}^{-a\left(1-r_{j}\right)} z_{11}}{1-z_{11}}\right)^{p}-1\right\} \leq \frac{1}{p} \frac{1}{(1-\rho)^{p}}\right|\left(\frac{1-\mathrm{e}^{-a\left(1-r_{j}\right)} z_{11}}{1-z_{11}}\right)^{p}-1 .
$$

Since

$$
\left|\frac{1-\mathrm{e}^{-a\left(1-r_{j}\right)} z_{11}}{1-z_{11}}-1\right|=\left|\frac{z_{11}}{1-z_{11}}\right| 1-\mathrm{e}^{-a\left(1-r_{j}\right)} \mid \leq \frac{1}{1-\rho}\left(1-\mathrm{e}^{-a\left(1-r_{j}\right)}\right) .
$$


But $1-\mathrm{e}^{-a\left(1-r_{j}\right)} \rightarrow 0$ as $j \rightarrow \infty$. Thus, $\left(\frac{1-\mathrm{e}^{-a\left(1-r_{j}\right)} z_{11}}{1-z_{11}}\right)^{p}-1$ converges to 0 uniformly on $E$. Therefore, $\left\{f_{j}\right\}$ converges to 0 uniformly on $E$ as $j \rightarrow \infty$. Thus, the sequence of functions $\left\{f_{j}(Z)\right\}$ defined by (4.10) satisfies the condition (II).

Now (4.8) and (4.9) mean that

$$
H_{\phi\left(Z^{j}\right)}\left(w^{j}, w^{j}\right)=(m, n)\left(A_{j}+B_{j}+C_{j}\right) \leq 3(m, n) A_{j} .
$$

Combining (4.7) and (4.16), we have

$$
\begin{aligned}
\left\|C_{\phi} f_{j}\right\|_{\beta^{q}} & \geq \operatorname{det}\left(I-Z^{j} \bar{Z}^{j^{\mathrm{T}}}\right)^{q} \frac{\left|\nabla\left(f_{j}\right)\left(\phi\left(Z^{j}\right)\right) J \phi\left(Z^{j}\right) u^{j}\right|}{H_{Z^{j}}^{1 / 2}\left(u^{j}, u^{j}\right)} \\
& \geq \varepsilon_{0}\left(1-r_{j}^{2}\right)^{p} \frac{\left|\nabla\left(f_{j}\right)\left(r_{j} E_{11}\right) w^{j}\right|}{H_{r_{j} E_{11}}^{1 / 2}\left(w^{j}, w^{j}\right)} \geq \frac{\varepsilon_{0}\left(1-r_{j}^{2}\right)^{p}}{\sqrt{3(m+n)}} \frac{\left|\frac{\partial f_{j}}{\partial z_{11}}\left(r_{j} E_{11}\right) w_{11}^{j}\right|}{\left|w_{11}^{j}\right| /\left(1-r_{j}^{2}\right)} \\
& \geq \frac{\varepsilon_{0}}{\sqrt{3(m+n)}}\left(1-\frac{\left(1-r_{j}\right)^{p+1} \mathrm{e}^{-a\left(1-r_{j}\right)}}{\left(1-\mathrm{e}^{-a\left(1-r_{j}\right)} r_{j}\right)^{p+1}}\right) .
\end{aligned}
$$

Since

$$
\lim _{j \rightarrow \infty}\left[1-\frac{\left(1-r_{j}\right)^{p+1} \mathrm{e}^{-a\left(1-r_{j}\right)}}{\left(1-\mathrm{e}^{-a\left(1-r_{j}\right)} r_{j}\right)^{p+1}}\right]=1-\left(\frac{1}{a+1}\right)^{p+1} \neq 0 .
$$

This proves that $\left\|C_{\phi} f_{j}\right\|_{\beta^{q}} \nrightarrow 0$ as $j \rightarrow \infty$, which means that the sequence of functions $\left\{f_{j}(Z)\right\}$ defined by (4.10) satisfies condition (III).

We can prove that the sequence of functions $\left\{f_{j}(Z)\right\}$ defined by (4.12) or (4.14) satisfies the conditions (I) - (III) by using the analogous method as above.

Part B: Now we assume that

$$
\phi\left(Z^{j}\right)=r_{j}^{(1)} E_{11}+r_{j}^{(2)} E_{22}, \quad j=1,2, \cdots
$$

It is clear that $1>r_{j}^{(1)} \geq r_{j}^{(2)} \geq 0$ and for $\phi\left(Z^{j}\right) \rightarrow \partial \Re_{I}(m, n)$ we can assume that $r_{j}^{(1)} \rightarrow 1$ and $r_{j}^{(2)} \rightarrow \lambda_{0}$ as $j \rightarrow \infty$, where $\lambda_{0} \leq 1$.

If $\lambda_{0}=1$, we can use the same methods as in Part A to construct a sequence of functions $\left\{f_{j}(Z)\right\}$ satisfying conditions (I)-(III).

Using formula (1.1), we have

$$
\begin{aligned}
& H_{\phi\left(Z^{j}\right)}\left(w^{j}, w^{j}\right)=(m+n) w^{j} \operatorname{diag}\left(\left(1-r_{j}^{(1)^{2}}\right)^{-1},\left(1-r_{j}^{(2)^{2}}\right)^{-1}, 1, \cdots, 1\right) \times \operatorname{diag}\left(\left(1-r_{j}^{(1)^{2}}\right)^{-1},\left(1-r_{j}^{(2)^{2}}\right)^{-1}, 1, \cdots, 1\right){\overline{w^{j}}}^{\mathrm{T}} \\
& =(m+n)\left[\frac{\left|w_{11}^{j}\right|^{2}}{\left(1-r_{j}^{(1)^{2}}\right)^{2}}+\frac{\left|w_{12}^{j}\right|^{2}+\left|w_{21}^{j}\right|^{2}}{\left(1-r_{j}^{(1)^{2}}\right)\left(1-r_{j}^{(2)^{2}}\right)}+\frac{\left|w_{22}^{j}\right|^{2}}{\left(1-r_{j}^{(2)}\right)^{2}}+\frac{1}{\left(1-r_{j}^{(1)^{2}}\right)}\left(\sum_{l=3}^{n}\left|w_{11}^{j}\right|^{2}+\sum_{k=3}^{m}\left|w_{k 1}^{j}\right|^{2}\right)\right. \\
& \left.+\frac{1}{\left(1-r_{j}^{(2)^{2}}\right)}\left(\sum_{l=3}^{n}\left|w_{2 l}^{j}\right|^{2}+\sum_{k=3}^{m}\left|w_{k 2}^{j}\right|^{2}\right)+\sum_{3 \leq k \leq m, 3 \leq l \leq n}\left|w_{k l}^{j}\right|^{2}\right] \text {. }
\end{aligned}
$$

Denote 


$$
\begin{aligned}
& A_{j}=\frac{\left|w_{11}^{j}\right|^{2}}{\left(1-r_{j}^{(1)^{2}}\right)^{2}}, \quad B_{j}=\frac{\left|w_{12}^{j}\right|^{2}+\left|w_{21}^{j}\right|^{2}}{\left(1-r_{j}^{(1)^{2}}\right)\left(1-r_{j}^{(2)^{2}}\right)}, \quad C_{j}=\frac{\left|w_{22}^{j}\right|^{2}}{\left(1-r_{j}^{(2)^{2}}\right)^{2}}, \\
& D_{j}=\frac{1}{\left(1-r_{j}^{(1)^{2}}\right)}\left(\sum_{l=3}^{n}\left|w_{11}^{j}\right|^{2}+\sum_{k=3}^{m}\left|w_{k 1}^{j}\right|^{2}\right), \quad E_{j}=\frac{1}{\left(1-r_{j}^{(2)^{2}}\right)}\left(\sum_{l=3}^{n}\left|w_{21}^{j}\right|^{2}+\sum_{k=3}^{m}\left|w_{k 2}^{j}\right|^{2}\right), \quad F_{j}=\sum_{3 \leq k \leq m, 3 \leq l \leq n}\left|w_{k l}^{j}\right|^{2} .
\end{aligned}
$$

Then,

$$
H_{\phi\left(Z^{j}\right)}\left(w^{j}, w^{j}\right)=(m+n)\left(A_{j}+B_{j}+C_{j}+D_{j}+E_{j}+F_{j}\right)
$$

We construct the sequence of functions $\left\{f_{j}\right\}$ according to the following six different cases.

Case 1. If for some $j$,

$$
\max \left(B_{j}, C_{j}, D_{j}, E_{j}, F_{j}\right) \leq A_{j}
$$

then set

$$
f_{j}(Z)=\frac{1}{p}\left(\frac{1}{\left(1-z_{11}\right)^{p}}-\frac{1}{\left(1-\mathrm{e}^{-a\left(1-r_{j}^{(1)}\right)} z_{11}\right)^{p}}\right)
$$

Case 2. If for some $j$,

$$
\max \left(A_{j}, C_{j}, D_{j}, E_{j}, F_{j}\right) \leq B_{j},
$$

then set

$$
f_{j}(Z)=\left(\mathrm{e}^{-i \theta_{12}^{j}} z_{12}+\mathrm{e}^{-i \theta_{21}^{j}} z_{21}\right)\left(\frac{1}{\left[\left(1-z_{11}\right)\left(1-z_{22}\right)\right]^{p+1 / 2}}-\frac{1}{\left[\left(1-\mathrm{e}^{-a\left(1-r_{j}^{(1)}\right)} z_{11}\right)\left(1-\mathrm{e}^{-a\left(1-r_{j}^{(2)}\right)} z_{22}\right)\right]^{p+1 / 2}}\right) .
$$

Case 3. If for some $j$,

$$
\max \left(A_{j}, B_{j}, D_{j}, E_{j}, F_{j}\right) \leq C_{j}
$$

then set

$$
f_{j}(Z)=\frac{1}{p}\left(\frac{1}{\left(1-z_{22}\right)^{p}}-\frac{1}{\left(1-\mathrm{e}^{-a\left(1-r_{j}^{(2)}\right)} Z_{22}\right)^{p}}\right) \text {. }
$$

Case 4. If for some $j$,

$$
\max \left(A_{j}, B_{j}, C_{j}, E_{j}, F_{j}\right) \leq D_{j}
$$

then set

$$
f_{j}(Z)=\left(\sum_{l=3}^{n} \mathrm{e}^{-i \theta_{11}^{j}} z_{1 l}+\sum_{k=3}^{m} \mathrm{e}^{-i \theta_{k 1}^{j}} z_{k 1}\right)\left(\frac{1}{\left(1-z_{11}\right)^{p+1 / 2}}-\frac{1}{\left(1-\mathrm{e}^{-a\left(1-r_{j}^{(1)}\right)} z_{11}\right)^{p+1 / 2}}\right) .
$$

Case 5. If for some $j$,

$$
\max \left(A_{j}, B_{j}, C_{j}, D_{j}, F_{j}\right) \leq E_{j},
$$

then set 


$$
f_{j}(Z)=\left(\sum_{l=3}^{n} \mathrm{e}^{-i \theta_{2 l}^{j}} z_{2 l}+\sum_{k=3}^{m} \mathrm{e}^{-i \theta_{k 2}^{j}} z_{k 2}\right)\left(\frac{1}{\left(1-z_{22}\right)^{p+1 / 2}}-\frac{1}{\left(1-\mathrm{e}^{-a\left(1-r_{j}^{(2)}\right)} z_{22}\right)^{p+1 / 2}}\right) .
$$

Case 6. If for some $j$,

$$
\max \left(A_{j}, B_{j}, C_{j}, D_{j}, E_{j}\right) \leq F_{j}
$$

then set

$$
f_{j}(Z)=\left(\sum_{3 \leq k \leq m, 3 \leq l \leq n} \mathrm{e}^{-i \theta_{k l}^{j}} z_{k l}\right)\left(\frac{1}{\left(1-z_{11}\right)^{p}}-\frac{1}{\left(1-\mathrm{e}^{-a\left(1-r_{j}^{(1)}\right)} z_{11}\right)^{p}}\right) .
$$

By using the same methods as in Part A, we can prove the sequences of functions $\left\{f_{j}(Z)\right\}$ defined by (4.18)-(4.23) satisfying conditions (I) - (III).

Now, as an example,we will prove that the sequence of functions $\left\{f_{j}(Z)\right\}$ defined by (4.19) satisfying the conditions (I) - (III).

For any $Z \in \Re_{I}(m, n)$, we have

$$
\left|z_{12}\right|^{2}<1-\left|z_{i i}\right|^{2}, \quad\left|z_{21}\right|^{2}<1-\left|z_{i i}\right|^{2}, \quad i=1,2
$$

Thus

$$
\begin{aligned}
& \left|\nabla f_{j}(Z)\right|=\left\{\sum_{1 \leq k \leq m, 1 \leq l \leq n}\left|\frac{\partial f_{j}}{\partial z_{k l}}(Z)\right|^{2}\right\}^{1 / 2} \\
& \leq\left(p+\frac{1}{2}\right)\left(\mathrm{e}^{-i \theta_{12}^{j}} z_{12}+\mathrm{e}^{-i \theta \theta_{21}^{j}} z_{21}\right)\left(\frac{1}{\left(1-z_{11}\right)^{p+\frac{3}{2}}\left(1-z_{22}\right)^{p+\frac{1}{2}}}-\frac{\mathrm{e}^{-a\left(1-r_{j}^{(1)}\right)}}{\left(1-\mathrm{e}^{-a\left(1-r_{j}^{(1)}\right)} z_{11}\right)^{p+\frac{3}{2}}\left(1-\mathrm{e}^{-a\left(1-r_{j}^{(2)}\right)} z_{22}\right)^{p+\frac{1}{2}}}\right) \mid \\
& +\left|\left(p+\frac{1}{2}\right)\left(\mathrm{e}^{-i \theta_{12}^{j}} z_{12}+\mathrm{e}^{-i \theta_{21}^{j}} z_{21}\right)\left(\frac{1}{\left(1-z_{11}\right)^{p+\frac{1}{2}}\left(1-z_{22}\right)^{p+\frac{3}{2}}}-\frac{\mathrm{e}^{-a\left(1-r_{j}^{(2)}\right)}}{\left(1-\mathrm{e}^{-a\left(1-r_{j}^{(1)}\right)} z_{11}\right)^{p+\frac{1}{2}}\left(1-\mathrm{e}^{-a\left(1-r_{j}^{(2)}\right)} z_{22}\right)^{p+\frac{3}{2}}}\right)\right| \\
& +2\left(\frac{1}{\left[\left(1-z_{11}\right)\left(1-z_{22}\right)\right]^{p+1 / 2}}-\frac{1}{\left[\left(1-\mathrm{e}^{-a\left(1-r_{j}^{(1)}\right)} z_{11}\right)\left(1-\mathrm{e}^{-a\left(1-r_{j}^{(2)}\right)} z_{22}\right)\right]^{p+1 / 2}}\right) \mid \\
& <\left(p+\frac{1}{2}\right)\left(\left|z_{12}\right|+\left|z_{21}\right|\right)\left(\frac{2}{\left(1-\left|z_{11}\right|\right)^{p+\frac{3}{2}}\left(1-\left|z_{22}\right|\right)^{p+\frac{1}{2}}}+\frac{2}{\left(1-\left|z_{11}\right|\right)^{p+\frac{1}{2}}\left(1-\left|z_{22}\right|\right)^{p+\frac{3}{2}}}\right)+\frac{4}{\left(1-\left|z_{11}\right|\right)^{p+\frac{1}{2}}\left(1-\left|z_{22}\right|\right)^{p+\frac{1}{2}}} \\
& \leq 4 \sqrt{2}\left(p+\frac{1}{2}\right)\left(\frac{1}{\left(1-\left|z_{11}\right|\right)^{p+1}\left(1-\left|z_{22}\right|\right)^{p+\frac{1}{2}}}+\frac{1}{\left(1-\left|z_{11}\right|\right)^{p+\frac{1}{2}}\left(1-\left|z_{22}\right|\right)^{p+1}}\right)+\frac{4}{\left(1-\left|z_{11}\right|\right)^{p+\frac{1}{2}}\left(1-\left|z_{22}\right|\right)^{p+\frac{1}{2}}} .
\end{aligned}
$$


By Lemma 2.1, we have

$$
\begin{aligned}
\operatorname{det} & \left(I-Z \bar{Z}^{\mathrm{T}}\right)^{p}\left\{\nabla f_{j}(Z) T^{-1}(Z, Z) \overline{\nabla f_{j}(Z)^{\mathrm{T}}}\right\}^{1 / 2} \\
\leq & \operatorname{det}\left(I-Z \bar{Z}^{\mathrm{T}}\right)^{p}\left|\nabla f_{j}(Z)\right|\left\{\operatorname{det} T^{-1}(Z, Z)\right\}^{1 / 2} \\
\leq & \frac{1}{\sqrt{m+n}}\left(1-\left|z_{11}\right|^{2}\right)^{p+\frac{m+n}{2}}\left(1-\left|z_{22}\right|^{2}\right)^{p+\frac{m+n}{2}} \\
& \times\left(\frac{4 \sqrt{2}\left(p+\frac{1}{2}\right)}{\left(1-\left|z_{11}\right|\right)^{p+1}\left(1-\left|z_{22}\right|\right)^{p+\frac{1}{2}}}+\frac{4 \sqrt{2}\left(p+\frac{1}{2}\right)}{\left(1-\left|z_{11}\right|\right)^{p+\frac{1}{2}}\left(1-\left|z_{22}\right|\right)^{p+1}}+\frac{4}{\left(1-\left|z_{11}\right|\right)^{p+\frac{1}{2}}\left(1-\left|z_{22}\right|\right)^{p+\frac{1}{2}}}\right) \\
\leq & \frac{2^{2 p+m+n+2}}{\sqrt{m+n}}\left(2 \sqrt{2}\left(p+\frac{1}{2}\right)+1\right) .
\end{aligned}
$$

It follows from Lemma 3.5 and (4.25) that $\left\|f_{j}\right\|_{\beta^{p}} \leq C$. This proves that the sequence of functions $\left\{f_{j}(Z)\right\}$ defined by (4.19) satisfy the condition (I).

Let $E$ be any compact subset of $\mathfrak{R}_{I}(m, n)$. Since there exists a $\rho \in(0,1)$ such that $1-\left|z_{i i}\right| \geq 1-\rho>0$, $i=1,2$ Thus

$$
\left|f_{j}(Z)\right| \leq \frac{2}{(1-\rho)^{2 p+1}}\left\{\left[\frac{\left(1-\mathrm{e}^{-a\left(1-r_{j}^{(1)}\right)} z_{11}\right)\left(1-\mathrm{e}^{-a\left(1-r_{j}^{(2)}\right)} z_{22}\right)}{\left(1-z_{11}\right)\left(1-z_{22}\right)}\right]^{p+1 / 2}-1\right\} .
$$

Since

$$
\left|\frac{1-\mathrm{e}^{-a\left(1-r_{j}^{(i)}\right)} z_{i i}}{1-z_{i i}}-1\right|=\left|\frac{z_{i i}}{1-z_{i i}}\right|\left|1-\mathrm{e}^{-a\left(1-r_{j}^{(i)}\right)}\right| \leq \frac{1}{1-\rho}\left(1-\mathrm{e}^{-a\left(1-r_{j}^{(i)}\right)}\right), \quad i=1,2 .
$$

So $1-\mathrm{e}^{-a\left(1-r_{j}^{(i)}\right)} \rightarrow 0$ as $j \rightarrow \infty$. Thus, $\left[\frac{\left(1-\mathrm{e}^{-a\left(1-r_{j}^{(1)}\right)} z_{11}\right)\left(1-\mathrm{e}^{-a\left(1-r_{j}^{(2)}\right)} z_{22}\right)}{\left(1-z_{11}\right)\left(1-z_{22}\right)}\right]^{p+1 / 2}-1$ converges to 0 uni-

formly on $E$. Therefore,the sequence of $\left\{f_{j}\right\}$ converges to 0 uniformly on $E$ as $j \rightarrow \infty$. Thus, the sequence of functions $\left\{f_{j}(Z)\right\}$ defined by (4.19) satisfies the condition (II).

For case 2,

$$
H_{\phi\left(Z^{j}\right)}\left(w^{j}, w^{j}\right) \leq 6(m+n) B_{j} .
$$

Combining (4.7) and (4.26), we have

$$
\begin{aligned}
& \left\|C_{\phi} f_{j}\right\|_{\beta^{q}} \geq \operatorname{det}\left(I-Z^{j} \bar{Z}^{j^{\mathrm{T}}}\right)^{q} \frac{\left|\nabla\left(f_{j}\right)\left(\phi\left(Z^{j}\right)\right) J \phi\left(Z^{j}\right) u^{j}\right|}{H_{Z^{j}}^{1 / 2}\left(u^{j}, u^{j}\right)} \geq \varepsilon_{0}\left(1-r_{j}^{(1)^{2}}\right)^{p}\left(1-r_{j}^{(2)^{2}}\right)^{p} \frac{\left|\nabla\left(f_{j}\right)\left(r_{j}^{(1)} E_{11}+r_{j}^{(2)} E_{22}\right) w^{j}\right|}{H_{r_{j}^{(1)} E_{11}+r_{j}^{(2)} E_{22}}\left(w^{j}, w^{j}\right)} \\
& \geq \frac{\varepsilon_{0}}{\sqrt{6(m+n)}}\left[1-\left(\frac{\left(1-r_{j}^{(1)}\right)\left(1-r_{j}^{(2)}\right)}{\left(1-\mathrm{e}^{-a\left(1-r_{j}^{(1)}\right)} r_{j}^{(1)}\right)\left(1-\mathrm{e}^{-a\left(1-r_{j}^{(2)}\right)} r_{j}^{(2)}\right)}\right)^{p+1 / 2}\right] .
\end{aligned}
$$

Since 


$$
\lim _{j \rightarrow \infty}\left[1-\left(\frac{\left(1-r_{j}^{(1)}\right)\left(1-r_{j}^{(2)}\right)}{\left(1-\mathrm{e}^{-a\left(1-r_{j}^{(1)}\right)} r_{j}^{(1)}\right)\left(1-\mathrm{e}^{-a\left(1-r_{j}^{(2)}\right)} r_{j}^{(2)}\right)}\right)^{p+1 / 2}\right]=1-\left(\frac{1}{a+1}\right)^{2 p+1} \neq 0 .
$$

This proves that $\left\|C_{\phi} f_{j}\right\|_{\beta^{q}} \nrightarrow 0$ as $j \rightarrow \infty$, which means that the sequence of functions $\left\{f_{j}(Z)\right\}$ defined by (4.19) satisfies condition (III).

If $\lambda_{0}<1$, then by Lemma 3.6, there exist $\Phi_{r_{j}^{(1)} E_{11}+r_{j}^{(2)} E_{22}}$ and $\Phi_{r_{j}^{(1)} E_{11}}$ in $\mathfrak{R}_{I}(m, n)$ such that

$$
\Phi_{r_{j}^{(1)} E_{11}+r_{j}^{(2)} E_{22}}\left(r_{j}^{(1)} E_{11}+r_{j}^{(2)} E_{22}\right)=0 \quad \text { and } \Phi_{r_{j}^{(1)} E_{11}}\left(r_{j}^{(1)} E_{11}\right)=0 \quad(j=1,2, \cdots)
$$

If we denote $\Psi^{(j)}(Z)=\left(\Phi_{r_{j}^{(1)}} E_{11}\right)^{-1} \circ \Phi_{r_{j}^{(1)} E_{11}+r_{j}^{(2)} E_{22}}$, then $\Psi^{(j)} \in \operatorname{Aut}\left(\Re_{I}(m, n)\right)$ and $\Psi^{(j)}\left(\phi\left(Z^{j}\right)\right)=\Psi^{(j)}\left(r_{j}^{(1)} E_{11}+r_{j}^{(2)} E_{22}\right)=r_{j}^{(1)} E_{11}=r_{j} E_{11}$, where $r_{j}=r_{j}^{(1)}$.

Denote $g_{j}=f_{j} \circ \Psi^{(j)}$, where the sequence of functions $\left\{f_{j}\right\}$ is the sequence obtained in Part A. We have

$$
H_{\phi\left(Z^{j}\right)}\left(w^{j}, w^{j}\right)=H_{\Psi^{(j)} \circ \phi\left(Z^{j}\right)}\left(J \Psi^{(j)}\left(\phi\left(Z^{j}\right)\right) w^{j}, J \Psi^{(j)}\left(\phi\left(Z^{j}\right)\right) w^{j}\right)=H_{r_{j} E_{11}}\left(v^{j}, v^{j}\right),
$$

where $w^{j}=J \phi\left(Z^{j}\right) u^{j}$ and $v^{j}=J \Psi^{(j)}\left(\phi\left(Z^{j}\right)\right) w^{j}$. Now (4.27) implies that

$$
\frac{\left|\nabla\left(g_{j}\right)\left(\phi\left(Z^{j}\right)\right) w^{j}\right|}{H_{\phi\left(Z^{j}\right)}^{1 / 2}\left(w^{j}, w^{j}\right)}=\frac{\left|\nabla\left(f_{j}\right)\left(r_{j} E_{11}\right) v^{j}\right|}{H_{r_{j} E_{11}}^{1 / 2}\left(v^{j}, v^{j}\right)}
$$

and

$$
\begin{aligned}
\left\|C_{\phi} g_{j}\right\|_{\beta^{q}} & \geq \operatorname{det}\left(I-Z \bar{Z}^{\mathrm{T}}\right)^{q} \frac{\left|\nabla\left(g_{j}\right)\left(\phi\left(Z^{j}\right)\right) J \phi\left(Z^{j}\right) u^{j}\right|}{H_{Z^{j}}^{1 / 2}\left(u^{j}, u^{j}\right)} \\
& \geq \varepsilon_{0} \operatorname{det}\left(I-\phi\left(Z^{j}\right) \overline{\phi\left(Z^{j}\right)^{\mathrm{T}}}\right)^{p} \frac{\left|\nabla\left(g_{j}\right)\left(\phi\left(Z^{j}\right)\right) w^{j}\right|}{H_{\phi\left(Z^{j}\right)}^{1 / 2}\left(w^{j}, w^{j}\right)} \\
& =\varepsilon_{0}\left(1-r_{j}^{(2)^{2}}\right)^{p}\left(1-r_{j}^{(1)^{2}}\right)^{p} \frac{\left|\nabla\left(f_{j}\right)\left(r_{j} E_{11}\right) v^{j}\right|}{H_{r_{j} E_{11}}^{1 / 2}\left(v^{j}, v^{j}\right)}
\end{aligned}
$$

It is clear that $\lim _{j \rightarrow \infty}\left(1-r_{j}^{(2)^{2}}\right)=1-\lambda_{0}^{2} \neq 0$, and combining the discussion in Part A, we can get that $\left\|C_{\phi} g_{j}\right\|_{\beta^{q}} \nrightarrow 0$ as $j \rightarrow \infty$; that means the sequence of functions $\left\{g_{j}\right\}$ satisfies condition (III).

We prove that the sequence of functions $\left\{g_{j}\right\}$ is a bounded sequence in $\beta^{p}\left(\Re_{I}(m, n)\right)$.

Since $\Psi^{j}(Z) \in \operatorname{Aut}\left(\Re_{I}(m, n)\right)$,

$$
\operatorname{det}\left(I-Z \bar{Z}^{\mathrm{T}}\right)^{p} Q_{g_{j}}(Z)=\operatorname{det}\left(I-Z \bar{Z}^{\mathrm{T}}\right)^{p} Q_{f_{j}}\left(\Psi^{(j)}(Z)\right) .
$$

So $\left\|g_{j}\right\|_{\beta^{p}}=\left\|f_{j}\right\|_{\beta^{p}}$ is bounded.

Next we prove that $\left\{g_{j}\right\}$ converges to 0 uniformly on any compact subset $E$ of $\mathfrak{R}_{I}(m, n)$. Let $\Psi^{(j)}(Z)=\left(\Psi_{l k}^{(j)}(Z)\right)_{1 \leq l \leq m, 1 \leq k \leq n}$, then by the definition of $\Psi^{(j)}$ and Lemma 3.6, we can get a calculation directly that 


$$
\Psi_{11}^{(j)}(Z)=z_{11}+r_{j}^{(2)} \frac{z_{12} z_{21}}{1-r_{j}^{(2)} z_{22}}
$$

It is clear that $\Psi_{11}^{(j)}(Z)$ converges uniformly to $\Psi_{11}(Z)=z_{11}+\lambda_{0} \frac{Z_{12} Z_{21}}{1-\lambda_{0} z_{22}}$ in $\mathfrak{R}_{I}(m, n)$.

Since $\lambda_{0}<1$ and $\lambda_{0} E_{11}+\lambda_{0} E_{22} \in \mathfrak{R}_{I}(m, n)$, there similarly exist $\Psi(Z)$ in $\operatorname{Aut}\left(\mathfrak{R}_{I}(m, n)\right)$ such that $\Psi\left(\lambda_{0} E_{11}+\lambda_{0} E_{22}\right)=\lambda_{0} E_{11}$, and the first component of $\Psi(Z)$ is $\Psi_{11}(Z)$. It is clear that $\Psi_{11}(Z)$ is holomorphic on $\mathfrak{R}_{I}(m, n)$. Let $M_{1}=\sup _{Z \in E}\left|\Psi_{11}(Z)\right|=\Psi_{11}\left(Z_{0}\right)$ for $Z_{0} \in E$. For $\Psi(Z) \in \operatorname{Aut}\left(\Re_{I}(m, n)\right)$, we know $M_{1}=\Psi_{11}\left(Z_{0}\right)<1$. We may choose $M_{0}>0$ such that $M_{1}<M_{0}<1$. Thus, for $j$ large enough, $\left|\Psi_{11}^{(j)}\left(Z_{0}\right)\right|<M_{0}$ and from this it follows that

$$
1-\left|\Psi_{11}^{(j)}(Z)\right|>1-M_{0}>0
$$

by the definition of $\left\{f_{j}(Z)\right\}, g_{j}(Z)=f_{j} \circ \Psi^{(j)}(Z)$ converges to 0 uniformly on $E$.

Hence $\left\{g_{j}(Z)\right\}$ satisfies conditions (I)--(III), and this contradicts the compactness of $C_{\phi}$.

Part C: Assume that

$$
\phi\left(Z^{j}\right)=\sum_{k=1}^{m} r_{j}^{(k)} E_{k k}, \quad j=1,2, \cdots
$$

where $1>r_{j}^{(1)} \geq r_{j}^{(2)} \geq \cdots \geq r_{j}^{(m)} \geq 0$. For $\phi\left(Z^{j}\right) \rightarrow \partial \Re_{I}(m, n)$ we may assume that $r_{j}^{(1)} \rightarrow 1, r_{j}^{(2)} \rightarrow \lambda_{2}, \cdots$ $r_{j}^{(m)} \rightarrow \lambda_{m}$ as $j \rightarrow \infty$, where $\lambda_{k} \leq 1, k=2,3, \cdots, m$.

Just as in Part B, we can use the same methods to prove the conclusion. And for $\lambda_{k}=1, k=2,3, \cdots, m$, we may only show the sequence of functions $\left\{f_{j}(Z)\right\}$ which satisfy the conditions (I) - (III) here.

Using formula (1.1), we have

$$
\begin{aligned}
H_{\phi\left(Z^{j}\right)}\left(w^{j}, w^{j}\right)=(m+n) & w^{j} \operatorname{diag}\left(\left(1-r_{j}^{(1)^{2}}\right)^{-1},\left(1-r_{j}^{(2)^{2}}\right)^{-1}, \cdots,\left(1-r_{j}^{(m)^{2}}\right)^{-1}\right) \\
& \times \operatorname{diag}\left(\left(1-r_{j}^{(1)^{2}}\right)^{-1},\left(1-r_{j}^{(2)^{2}}\right)^{-1}, \cdots,\left(1-r_{j}^{(m)^{2}}\right)^{-1}, 1, \cdots, 1\right){\overline{w^{j}}}^{\mathrm{T}} \\
= & (m+n)\left[\sum_{k=1}^{m} \frac{\left|w_{k k}^{j}\right|^{2}}{\left(1-r_{j}^{(k)^{2}}\right)^{2}}+\sum_{1 \leq k<l \leq m} \frac{\left|w_{k l}^{j}\right|^{2}+\left|w_{l k}^{j}\right|^{2}}{\left(1-r_{j}^{(k)^{2}}\right)\left(1-r_{j}^{(l)}\right)^{2}}+\sum_{k=1}^{m} \frac{\sum_{l=m+1}^{n}\left|w_{k l}^{j}\right|^{2}}{1-r_{j}^{(k)^{2}}}\right] .
\end{aligned}
$$

Denote

$$
\begin{aligned}
& A_{j}^{(k)}=\frac{\left|w_{k k}^{j}\right|^{2}}{\left(1-r_{j}^{(k)^{2}}\right)^{2}}, \quad k=1, \cdots, m, \quad B_{j}^{(k l)}=\frac{\left|w_{k l}^{j}\right|^{2}+\left|w_{l k}^{j}\right|^{2}}{\left(1-r_{j}^{(k)^{2}}\right)\left(1-r_{j}^{(l)}\right)}, \quad 1 \leq k<l \leq m, \\
& C_{j}^{(k)}=\frac{\sum_{l=m+1}^{n}\left|w_{k l}^{j}\right|^{2}}{1-r_{j}^{(k)^{2}}}, \quad k=1, \cdots, m .
\end{aligned}
$$

then,

$$
H_{\phi\left(Z^{j}\right)}\left(w^{j}, w^{j}\right)=(m+n)\left(\sum_{k=1}^{m} A_{j}^{(k)}+\sum_{1 \leq k<l \leq m} B_{j}^{(k l)}+\sum_{k=1}^{m} C_{j}^{(k)}\right) .
$$

We construct the sequence of functions $\left\{f_{j}\right\}$ according to the following three different cases.

Case 1. If for some $j$,

$$
\max \left(A_{j}^{(i)}, B_{j}^{(s t)}, C_{j}^{(i)}\right) \leq A_{j}^{(k)}, \quad i, k=1, \cdots, m, \quad 1 \leq s<t \leq m,
$$


then set

$$
f_{j}^{(k)}(Z)=\frac{1}{p}\left(\frac{1}{\left(1-z_{k k}\right)^{p}}-\frac{1}{\left(1-\mathrm{e}^{-a\left(1-r_{j}^{(k)}\right)} z_{k k}\right)^{p}}\right), \quad k=1, \cdots, m .
$$

Case 2. If for some $j$,

$$
\max \left(A_{j}^{(i)}, B_{j}^{(s t)}, C_{j}^{(i)}\right) \leq B_{j}^{(k l)}, \quad i=1, \cdots, m, \quad 1 \leq s<t \leq m, \quad 1 \leq k<l \leq m,
$$

then set

$$
f_{j}^{(k l)}(Z)=\left(\mathrm{e}^{-i \theta_{k l}^{j}} z_{k l}+\mathrm{e}^{-i \theta \theta_{l k}^{j}} z_{l k}\right)\left(\frac{1}{\left[\left(1-z_{k k}\right)\left(1-z_{l l}\right)\right]^{p+1 / 2}}-\frac{1}{\left[\left(1-\mathrm{e}^{-a\left(1-r_{j}^{(k)}\right)} Z_{k k}\right)\left(1-\mathrm{e}^{-a\left(1-r_{j}^{(l)}\right)} z_{l l}\right)\right]^{p+1 / 2}}\right),
$$

Case 3. If for some $j$,

$$
\max \left(A_{j}^{(i)}, B_{j}^{(s t)}, C_{j}^{(i)}\right) \leq C_{j}^{(k)}, \quad i, k=1, \cdots, m, \quad 1 \leq s<t \leq m,
$$

then set

$$
f_{j}^{(k)}(Z)=\left(\sum_{l=m+1}^{n} \mathrm{e}^{-i \theta_{k l}^{j}} z_{k l}\right)\left(\frac{1}{\left(1-z_{k k}\right)^{p+1 / 2}}-\frac{1}{\left(1-\mathrm{e}^{-a\left(1-r_{j}^{(k)}\right)} z_{k k}\right)^{p+1 / 2}}\right), \quad k=1, \cdots, m
$$

Using the same methods as in Part A and Part B, we can prove the sequences of functions $\left\{f_{j}(Z)\right\}$ defined by (4.29)-(4.31) satisfying conditions (I) - (III).

Part D: In the general situation. For $\phi\left(Z^{j}\right) \in \mathfrak{R}_{I}(m, n)$, there exist an $m \times m$ unitary matrix $P_{j}$ and an $n \times n$ unitary matrix $Q_{j}$ such that

$$
P_{j}\left(\phi\left(Z^{j}\right)\right) Q_{j}=\sum_{k=1}^{m} r_{j}^{(k)} E_{k k} .
$$

We may assume that $P_{j} \rightarrow P$ and $Q_{j} \rightarrow Q$ as $j \rightarrow \infty$. Let $P_{j}=\left(p_{j}^{k l}\right)$ and $P=\left(p^{k l}\right) ; P_{j} \rightarrow P$ means that $p_{j}^{k l} \rightarrow p^{k l}$ as $j \rightarrow \infty$ for any $1 \leq k, l \leq m$. Let $\Psi^{j}(Z)=P_{j} Z Q_{j}$ and $\Psi(Z)=P Z Q$ for $Z \in \mathfrak{R}_{I}(m, n)$. Of course, $P$ is an $m \times m$ unitary matrix, $Q$ is an $n \times n$ unitary matrix, and $\left\{\Psi^{(j)}(Z)\right\}$ converges uniformly to $\Psi(Z)$ on $\mathfrak{R}_{I}(m, n)$.

Let $g_{j}(Z)=f_{j}\left(\Psi^{j}(Z)\right), j=1,2, \cdots$ where the sequence of $\left\{f_{j}\right\}$ are the functions obtained in Part C. From the same discussion as that in Part B, we know that $\left\{g_{j}(Z)\right\}$ satisfies conditions (I) and (III). For the compact subset $E \subset \mathfrak{R}_{I}(m, n), \Psi(E)$ is also a compact subset of $\mathfrak{R}_{I}(m, n)$, so we can choose an open subset $D_{1}$ of $\mathfrak{R}_{I}(m, n)$ such that $\Psi(E) \subset D_{1} \subset \overline{D_{1}} \subset \mathfrak{R}_{I}(m, n)$. Since $\left\{\Psi^{(j)}(Z)\right\}$ converges uniformly to $\Psi(Z)$ on $\mathfrak{R}_{I}(m, n)$, it follows that $\Psi^{j}(E) \subset D_{1}$ as $j \rightarrow \infty$. Since $\left\{f_{j}(Z)\right\}$ tends to 0 uniformly on $\overline{D_{1}}$, we know $\left\{g_{j}(Z)\right\}$ tends to 0 uniformly on $E$. Thus, $\left\{g_{j}\right\}$ satisfies condition (II). 


\section{Acknowledgements}

We thank the Editor and the referee for their comments. Research is funded by the National Natural Science Foundation of China (Grant No. 11171285) and the Postgraduate Innovation Project of Jiangsu Province of China (CXLX12-0980).

\section{References}

[1] Ramos-Fernández, J.C. (2011) Composition Operators between $\mu$-Bloch Spaces. Extracta Mathematicae, $26,75-88$.

[2] Wolf, E. (2011) Weighted Composition Operators between Weighted Bloch Type Spaces. Bulletin de la Société Royale des Sciences de Liège, 80, 806-816.

[3] Dai, J.N. (2012) Compact Composition Operators on the Bloch Space of the Unit Ball. Journal of Mathematical Analysis and Applications, 386, 294-299. http://dx.doi.org/10.1016/j.jmaa.2011.07.067

[4] Li, J.F. (2010) Composition Operators from p-Bloch Spaces to Little q-Bloch Spaces on the Unit Ball of $C^{n}$. Acta Mathematica Sinica (Series B), 30, 1013-1020.

[5] Zhang, M.Z. and Xu, W. (2007) Composition Operators on $\alpha$-Bloch Spaces of the Unit Ball. Acta Mathematica Sinica (Series B), 23, 1991-2002.

[6] Zhang, X.J. and Li, J.X. (2009) Weighted Composition Operators Between $\mu$-Bloch Spaces over the Unit Ball of $C^{n}$. (Chinese). Acta Mathematica Sinica (Series A), 29, 573-583.

[7] Zhou, Z.H. and Zeng, H.G. (2003) Composition Operators between p-Bloch Space and q-Bloch Space in the Unit Ball. Progr. Progress in Natural Science (Engl. Ed.), 13, 233-236.

[8] Stevo, S. (2008) Norm of Weighted Composition Operators from Bloch Space to $H_{\mu}^{\infty}$ on the Unit Ball. Ars Combinatoria, 88, 125-127.

[9] Tang, X.M. and Zhang, R.J. (2013) Weighted Composition Operator from Bloch-Type Space to $H^{\infty}$ Space on the Unit Ball. Mathematical Inequalities and Applications, 16, 289-299. http://dx.doi.org/10.7153/mia-16-22

[10] Li, S.X. and Zhu, X.L. (2004) Essential Norm of Weighted Composition Operator between $\alpha$-Bloch Space and $\beta$-Bloch Space in Polydiscs. International Journal of Mathematics and Mathematical Sciences, 69-72, 3941-3950.

[11] Zhou, Z.H. and Shi, J.H. (2001) Compact Composition Operators on the Bloch Space in Polydiscs. Science in China Series A, 44, 286-291. http://dx.doi.org/10.1007/BF02878708

[12] Zhou, Z.H. and Wei, Z.Q. (2005) Weighted Composition Operators on the Bloch Space in Polydiscs (Chinese). Journal of Mathematics (Wuhan University), 25, 435-440.

[13] Allen, R.F. and Colonna, F. (2010) Weighted Composition Operators on the Bloch Space of a Bounded Homogeneous Domain. In: Operator Theory: Advances and Applications, Volume 1. Operators, Matrices and Analytic Functions, Oper. Theory Adv. Appl., 202, Birkhäuser Verlag, Basel, 11-37.

[14] Allen, R.F. and Colonna, F. (2009) Multiplication Operators on the Bloch Space of Bounded Homogeneous Domains. Computational Methods and Function Theory, 9, 679-693. http://dx.doi.org/10.1007/BF03321751

[15] Deng, F.W. and Ouyang, C.H. (2006) Bloch Spaces on Bounded Symmetric Domains in Complex Banach Spaces. Science in China Series A, 49, 1625-1632. http://dx.doi.org/10.1007/s11425-006-2050-0

[16] Shi, J.H. and Luo, L. (2001) Composition Operators on the Bloch Space of Several Complex Variables (Chinese). Acta Mathematica Sinica (Chinese Series), 44, 1-10.

[17] Timony, R.M. (1980) Bloch Functions in Several Complex Variables, I. Bulletin of the London Mathematical Society, 12, 241-267. http://dx.doi.org/10.1112/blms/12.4.241

[18] Zhou, Z.H. and Shi, J.H. (2002) Compactness of Composition Operators on the Bloch Space in Classical Bounded Symmetric Domains. Michigan Mathematical Journal, 50, 381-405. http://dx.doi.org/10.1307/mmj/1028575740

[19] Lu, Q.K. (1963) The Classical Manifolds and the Classical Domains (Chinese). Shanghai Scientific and Technical Publishers, Shanghai.

[20] Pan, W.Q. (2012) Composition Operators between p-Bloch Space and q-Bloch Space in the First Classical Bounded Symmetric Domain. Master's Thesis, Jiangsu Normal University, Xuzhou.

[21] Kuang, J.C. (2004) Applied Inequalities (Chinese). 3nd Edition, Shandong Science and Technology Press, Shandong. 